\title{
A Single-Center Experience of Antimicrobial Resistance Patterns in Pediatric Urinary Tract Infection
}

\author{
Saliha Senel ${ }^{a}$ Candemir Karacan $^{\text {a }}$ Nilgun Erkek $^{\mathrm{a}}$ Nese Gol ${ }^{\mathrm{b}}$ \\ Departments of a Pediatrics and ${ }^{b}$ Microbiology, Dr. Sami Ulus Children's Health and Diseases Training and \\ Research Hospital, Ankara, Turkey
}

\section{Key Words}

Antimicrobial resistance $\cdot$ Pediatric infections $\cdot$ Resistance patterns - Susceptibility testing - Urinary tract infection • Uropathogens

\begin{abstract}
Objective: To assess the prevalence of urinary tract pathogens and their resistance patterns against antimicrobial agents in a single center. Patients and Methods: In children $<16$ years of age admitted for urinary tract infection (UTI) to the Dr. Sami Ulus Teaching and Training Hospital from January 2004 to December 2008, positive urine cultures were reviewed. Results: A total of 3,485 positive urine cultures were identified, of which 2,379 (68\%) were from females and 106 (32\%) from males. Their mean age was $63.5 \pm 40.7$ months. Escherichia coli was the most common causative agent both in total and among different age groups. Ampicillin had the highest resistance rate from all the pathogens isolated (63.8\%), followed by piperacillin (51.8\%) and trimethoprimsulfamethoxazole (TMP-SMX; 48.6\%). Cephalotin also had a high resistance rate (32.7\%). The least resistance was for imipenem, amikacin, netilmicin and ciprofloxacin $(0.13,1.7,2.4$ and $7.5 \%$, respectively). None of the Klebsiella and Pseudomonas isolates were resistant to imipenem. None of the Staphylococcus aureus isolates were resistant to teicoplanin and
\end{abstract}

\section{KARGER}

Fax +41613061234

E-Mail karger@karger.ch

www.karger.com
(C) 2010 S. Karger AG, Basel

1011-7571/10/0195-0359\$26.00/0

Accessible online at:

www.karger.com/mpp vancomycin. Vancomycin-resistant Enterococcus spp. were isolated from two cultures. Conclusion: E. coli was the most common causative agent of UTI in children. Ampicillin, TMPSMX or cephalothin and piperacillin had the highest resistance rates against urinary tract pathogens in our center.

Copyright $\odot 2010$ S. Karger AG, Basel

\section{Introduction}

Urinary tract infection (UTI) is one of the most common infections in children $[1,2]$, and early diagnosis and early antimicrobial therapy significantly reduce serious consequences of UTI, such as renal failure and hypertension [2]. Children with UTI are usually treated empirically. Resistance of urinary tract pathogens to antimicrobial agents is an important problem in many countries, with increasing numbers of therapeutic failure after empiric treatment [3]. Resistance patterns to antimicrobial agents vary according to the geographic region [4]; consequently, the choice of antimicrobial empiric treatment should not only be based on the most likely urinary tract pathogens but also on their updated local resistance patterns [5]. Hence the aim of this study was to assess the most likely urinary tract pathogens and their resistance patterns against antimicrobial agents in our center. 


\section{Patients and Methods}

We reviewed all urine cultures from January 2004 to December 2008 from children $<16$ years of age admitted to the Dr. Sami Ulus Children's Health and Diseases Training and Research Hospital. In total, 3,485 positive urine cultures and antibiograms from children with UTI were identified. The study was approved by the Local Ethics Board.

All urine samples were obtained by midstream clean catch, transurethral bladder catheterization and suprapubic aspiration. Sterile urine bag samples were excluded. All urine samples were processed on blood agar and eosin methylene blue by standard loop method and incubated at $37^{\circ} \mathrm{C}$ overnight. A positive urine culture was defined as growth of a single pathogen of $>10^{5} \mathrm{col}-$ ony-forming units (CFU)/ml in a midstream urine sample, $>10^{4}$ $\mathrm{CFU} / \mathrm{ml}$ in a urine sample obtained by catheterization [6] and any number of colonies in a sample obtained by suprapubic aspiration [5].

Only the first positive urine culture obtained per patient on admission was included in the analysis to eliminate any possibility of recurrence and nosocomial infection. The polymicrobial cultures and cultures with multidrug-resistant uropathogens were not included in the analysis. Cultures with common contaminants, including coagulase-negative staphylococci and $\alpha$-hemolytic streptococci, and cultures with Candida growth were excluded. Bacterial identification and the determination of sensitivity and resistance patterns of these microorganisms were assessed by the criteria established by the Clinical Laboratory Standards Institute (formerly National Committee for Clinical Laboratory Standards) protocol [7]. For each UTI patient, age, gender, causative pathogen and antimicrobial resistance to a panel of antimicrobials were recorded. Data were entered into Microsoft Excel and analyzed. Results were reported as percentages. The mean age ( \pm SD) was determined. Median resistance to antimicrobial agents was calculated for each causative pathogen.

\section{Results}

A total of 3,485 positive urine cultures were identified, $2,379(68 \%)$ cultures were from females and 1,106 (32\%) from males. The female/male ratio was 2.1/1.

Of the positive urine cultures, $686(19.6 \%)$ were from infants (aged 0-24 months), 999 (28.7\%) from preschool children ( $2-5$ years), 1,783 (51.2\%) from school-age children (5-12 years) and $17(0.5 \%)$ from children $>12$ years. The mean age was $63.5 \pm 40.7$ months (range 0-192 months). Sterile urine bag samples were excluded from this study, and the majority of the cultures were from children $>2$ years. However, urine samples from infants aged $0-2$ years (table 1) were obtained by transurethral bladder catheterization and suprapubic aspiration. Escherichia coli was the most common causative agent $(2,473$ isolates; $71 \%$ ) both in total and among different age groups (table 1), followed by Proteus mirabilis (299 iso-
Table 1. Percentage of $E$. coli and various drug resistance rates against $E$. coli according to age groups

$0-2$ years $2-5$ years $5-12$ years $>12$ years

\begin{tabular}{lllll}
\hline $\begin{array}{l}\text { E. coli } \\
\quad \%\end{array}$ & 57 & 68 & 80 & 53 \\
$\mathrm{n} /$ total n & $391 / 686$ & $679 / 999$ & $1,483 / 1,783$ & $9 / 17$ \\
\hline
\end{tabular}

Resistance, \%

Amikacin

Netilmicin

4.2

4.2
4.6

Gentamicin

11.8

Ciprofloxacin

Cephazolin

7.5

Cephalotin

24

Cefuroxime

Ceftazidime

39.7

17.3

Ceftriaxone

Cefoperazone

Cefepime

Ampicillin

13.5

14.8

19

13.5

Amoxicillin-

$$
69.2
$$

clavulanate

Ampicillin-

sulbactam

TMP-SMX

Piperacillin

Imipenem

19

Aztreonam

\section{0}

\section{5}

62.4

62.4
-
13.9

13.9

\section{9}

1.5

7.7

7.4

17

34.6

10.9

7.8

8.4

14

8

71

21

38

55.5

63

$-=$ No resistance.

lates; 8.6\%), Klebsiella spp. (233 isolates; 6.7\%), Enterococcus spp. (221 isolates; 6.3\%), Morganella morganii (39 isolates; 1.1\%), Staphylococcus aureus (38 isolates; 1.1\%), Pseudomonas spp. (35 isolates; 1.1\%) and others.

From all the pathogens isolated, ampicillin had the highest resistance rate, followed by piperacillin and trimethoprim-sulfamethoxazole (TMP-SMX; 63.8, 51.8 and $48.6 \%$, respectively) and imipenem, amikacin, netilmicin and ciprofloxacin had the least resistance $(0.13,1.7$, 2.4 and $7.5 \%$, respectively; table 2 ).

E. coli presented high resistance to ampicillin, followed by piperacillin and TMP-SMX (65.7, 56.5 and $50.8 \%$, respectively). Resistance against cephalotin was also high (32.7\%). Imipenem was the most active agent against $E$. coli with an extremely low resistance rate $(0.08 \%)$. Only two of the E. coli isolates were found to be resistant to imipenem. E. coli presented low resistance to amikacin, netilmicin, gentamicin and ciprofloxacin (1.6, $2,8.5$ and $8.8 \%$, respectively; table 2). Urine cultures of 
Table 2. Resistance rates (\%) of the most frequent urinary tract pathogens against tested antibiotics

\begin{tabular}{|c|c|c|c|c|c|c|c|}
\hline Antibiotic & $\begin{array}{l}\text { E. } \\
\text { coli }\end{array}$ & $\begin{array}{l}K . \\
\text { oxytoco }\end{array}$ & $\begin{array}{l}\text { K. } \\
\text { pneumoniae }\end{array}$ & $\begin{array}{l}\text { M. } \\
\text { morganii }\end{array}$ & $\begin{array}{l}\text { Proteus } \\
\text { spp. }\end{array}$ & $\begin{array}{l}\text { Pseudomonas } \\
\text { spp. }\end{array}$ & $\begin{array}{l}\text { Overall } \\
\text { resistance }\end{array}$ \\
\hline Amikacin & 1.6 & 1.7 & 3.5 & - & 1.0 & - & 1.70 \\
\hline Netilmicin & 2.0 & 1.8 & 5.7 & - & 2.8 & 5.8 & 2.41 \\
\hline Gentamicin & 8.5 & 5.2 & 10.3 & 2.6 & 10.1 & 8.6 & 8.70 \\
\hline Ciprofloxacin & 8.8 & 1.7 & 2.3 & - & 1.0 & 2.8 & 7.52 \\
\hline Cephazolin & 17.2 & 62.5 & 36.6 & 94.8 & 45.3 & NT & 23.68 \\
\hline Cephalotin & 32.6 & 61.4 & 47.1 & 94.9 & 33.9 & NT & 35.58 \\
\hline Cefuroxime & 11.3 & 45.6 & 23.8 & 71.1 & 21.1 & NT & 14.83 \\
\hline Ceftazidime & 9.3 & 35.0 & 20.4 & - & 1.4 & 14.3 & 9.65 \\
\hline Ceftriaxone & 9.6 & 40.7 & 20.8 & - & 3.1 & 26.5 & 10.24 \\
\hline Cefoperazone & 14.7 & 52.6 & 28.6 & 2.7 & 6.6 & 3.1 & 15.26 \\
\hline Cefepime & 8.9 & 35.7 & 18.8 & - & 1.4 & 14.3 & 9.22 \\
\hline Ampicillin & 65.7 & 96.5 & 93.1 & 87.2 & 58.5 & NT & 63.85 \\
\hline Amoxicillin-clavulanate & 18.1 & 33.3 & 36.6 & 84.2 & 9.7 & NT & 20.20 \\
\hline Ampicillin-sulbactam & 34.4 & 63.1 & 55.4 & 34.2 & 11.9 & NT & 34.12 \\
\hline TMP-SMX & 50.7 & 16.1 & 22.7 & 41.1 & 60.6 & 97.1 & 48.57 \\
\hline Piperacillin & 56.5 & 63.1 & 61.7 & 12.8 & 21.1 & 2.8 & 51.77 \\
\hline Imipenem & 0.08 & - & - & - & 0.3 & - & 0.13 \\
\hline Aztreonam & 9.5 & 38.6 & 19.1 & - & 1.7 & 15.2 & 9.9 \\
\hline
\end{tabular}

children aged between $2-5$ and 5-12 years constituted $80 \%$ of all, and antibiotic resistance patterns against $E$. coli were comparable to total resistance data. Although antimicrobial resistance against $E$. coli was high in children $>12$ years, the results were ignored because there were only 9 samples in this age group (table 1).

$P$. mirabilis had significant resistance rates to TMPSMX, ampicillin and cephazolin (60.7, 58.5 and 45.3\%, respectively), with low resistance rates to amikacin, ciprofloxacin and netilmicin (1, 1 and $2.81 \%$, respectively). Only one isolate was found to be resistant to imipenem (table 2).

Klebsiella spp. had considerable resistance rates to ampicillin (93\% for Klebsiella pneumoniae and 96.5\% for Klebsiella oxytoco). None of these isolates was resistant to imipenem. Least resistance was observed to amikacin (3.5\% for K. pneumoniae and $1.7 \%$ for K. oxytoco) and ciprofloxacin (2.3\% for K. pneumoniae and $1.8 \%$ for $K$. oxytoco; table 2).

P. aeruginosa was most resistant to ceftriaxone (26.5\%). None of the isolates was resistant to imipenem and amikacin, and resistance to ciprofloxacin (2.7\%), piperacillin $(2.8 \%)$ and cefoperazone (3.1) was low (table 2).

None of the $M$. morganii isolates was resistant to imipenem, cefepime, amikacin and ciprofloxacin (table 2).
S. aureus was most resistant to penicillin G $(86.8 \%)$, but resistance to ciprofloxacin was low (8.6\%), with no resistance to teicoplanin and vancomycin (data not shown).

Vancomycin-resistant Enterococcus spp. were isolated from 2 cultures (data not shown).

\section{Discussion}

UTI are generally mediated by Gram-negative bacteria among which E. coli is the principal pathogen followed in frequency by Klebsiella spp., Proteus spp., P. aeruginosa, Enterococcus spp., Serratia spp. and S. aureus [8]. In the present study, E. coli was the most prevalent pathogen both in total and among different age groups, consistent with previous studies $[4,6,8]$. In this and previous reports $[2,4,6]$, the majority of cultures were from females.

According to WHO guidelines, TMP-SMX and ampicillin are the first choice for treatment of pediatric UTI [9], but resistance to TMP-SMX and ampicillin has progressively increased in the last decade $[3,4,6]$. E. coli showed high resistance to ampicillin and TMP-SMX in this study. Almost $65 \%$ of the uropathogens were resistant to ampicillin and nearly half of them were resistant 
to TMP-SMX, indicating that empiric treatment with ampicillin and TMP-SMX is inadequate for UTI in children in our center. The resistance rates to ampicillin and TMP-SMX reported in the present study were similar to other drug resistance studies in our country $[4,10]$, but much higher than those published in Europe and North America $[3,11,12]$ and lower than those reported in other countries, e.g. Iran, Taiwan, the Arab world and India $[2,6,13-15]$.

It has been suggested that children with acute pyelonephritis could effectively be treated with oral antibiotics, such as cefixime, ceftibuten and amoxicillin-clavulanate, with or without initial intravenous therapy [16]. Based on our results of $15 \%$ resistance to cefuroxime and $20 \%$ resistance to amoxicillin-clavulanate, we recommend these drugs for common treatment of primary, uncomplicated UTI in children in our center.

The prevalence of resistance to injectable antimicrobial agents was lowest for imipenem, followed by amikacin and ciprofloxacin in our patients, in agreement with previous studies $[4,11]$. Nearly all pathogens were found to be sensitive to imipenem. It is highly stable against $\beta$ lactamase and has the unusual property of having a postantimicrobial effect on Gram-negative bacteria. Due to its small molecular size, it can overcome the poor permeability of $\beta$-lactams by efficient penetration through the outer-membrane porin $\mathrm{D}$ [15].

Aminoglycosides do not negatively impact the intestinal flora, and resistance against aminoglycosides has remained very low (approximately 1-2\%) [17]. The prevalence of resistance against amikacin and netilmicin was very low in our patients, confirming the findings of recently published studies $[6,10,17]$. Hence, amikacin or netilmicin monotherapy could be used for the effective and safe management of UTI requiring parenteral treatment in our center.

There are limitations against the routine use of fluoroquinolones in pediatric patients due to the discovery of arthropathies in animal models [18]. However, they can be used in complicated UTI as they decrease hospitalization time [18]. In longitudinal studies, prevalence of ciprofloxacin resistance increased over time $[3,19]$, reaching almost 20\% in countries such as Spain and Portugal [3]. The prevalence of ciprofloxacin resistance seems to be higher in Latin America $[3,18]$. In our patients, the prevalence of ciprofloxacin resistance was higher than in reports from North America and European countries [3].

Resistance to other antimicrobial agents like cephalosporins is an emerging issue in children with UTI [4]. One possible reason for this is the widespread use of these agents in the pediatric population for otitis media and pharyngitis. Widespread cephalosporin use has been associated with the emergence of resistance to extendedspectrum $\beta$-lactamase, i.e. $\beta$-lactamases that hydrolyze extended-spectrum cephalosporins with an oxyimino side chain. These include ceftazidime, ceftriaxone, cefotaxime and the oxyimino monobactam aztreonam [4, 20]. In this study, the overall prevalence of resistance to ceftriaxone was almost $10 \%$ along with cefepim, ceftazidime and aztreonam, being in contrast to reports from European countries. In a study in Switzerland, no resistance was identified for third-generation cephalosporins [21], and in a study in Austria, resistance to cephalosporins was considerably low [11]. In a study in Jordan, the prevalence of resistance to these agents was higher than in our study, but we could not find a valid explanation for this discrepancy [14]. In countries like Iran and Brazil, the prevalence of resistance to cephalosporins was comparable with our isolates $[2,6]$. This class should be used with caution and should not be used if microbiological documentation is available in our country.

Almost $60 \%$ of E. coli and Klebsiella isolates were resistant to piperacillin. The elevated resistance to piperacillin (60\%) in this study was almost exclusively due to the primary resistance of E. coli and Klebsiella spp., as described in the literature [4].

The inappropriate usage of antimicrobial agents, poor hygienic conditions and overcrowded living conditions may result in the selection of resistant bacterial strains causing therapeutic failure $[3,5]$. The high resistance rates to antimicrobial agents in this study may be explained by the easy access to antimicrobial agents even in the absence of a prescription since antimicrobial drugs can be purchased in our country without a prescription. A population with a high percentage of young individuals with low socioeconomic status may contribute as another factor since our hospital is in the suburbs of Ankara, the capital of Turkey.

\section{Conclusion}

E. coli was the most common causative agent of UTI in children, and ampicillin, TMP-SMX or cephalothin and piperacillin had the highest resistance rates against uropathogens in our center. 


\section{References}

$>1$ Dimitrov TS, Udo EE, Emara M, Awni F, Passadilla R: Etiology and antibiotic susceptibility patterns of community-acquired urinary tract infections in a Kuwait hospital. Med Princ Pract 2004;13:334-339.

$\checkmark 2$ Sharifian M, Karimi A, Tabatabaei SR, Anvaripour N: Microbial sensitivity pattern in urinary tract infections in children: a single center experience of 1,177 urine cultures. Jpn J Infect Dis 2006;59:380-382.

3 Erb A, Stürmer T, Marre R, Brenner H: Prevalence of antibiotic resistance in Escherichia coli: overview of geographical, temporal, and methodological variations. Eur J Clin Microbiol Infect Dis 2007;26:83-90.

4 Catal F, Bavbek N, Bayrak O, Karabel M, Karabel D, Odemis E, Uz E: Antimicrobial resistance patterns of urinary tract pathogens and rationale for empirical therapy in Turkish children for the years 2000-2006. Int Urol Nephrol 2008, E-pub ahead of print.

$>5$ Goldraich NP, Manfroi A: Febrile urinary tract infection: Escherichia coli susceptibility to oral antimicrobials. Pediatr Nephrol 2002; 17:173-176.

6 Guidoni EB, Berezin EN, Nigro S, Santiago NA, Benini V, Toporovski J: Antibiotic resistance patterns of pediatric community-acquired urinary infections. Braz J Infect Dis 2008;12:321-323.

7 National Committee for Clinical Laboratory Standards: Performance Standards for Antimicrobial Susceptibility Testing. Twelfth Informational Supplement. NCCLS document M100-S12. Wayne, National Committee for Clinical Laboratory Standards, 2002.
8 Wiswell TE: The prepuce, urinary tract infections, and the consequences. Pediatrics 2000;105:860-862.

-9 Wolff O, Maclennan C: Evidence behind the WHO guidelines: hospital care for children: what is the appropriate empiric antimicrobial agent therapy in uncomplicated urinary tract infections in children in developing countries? J Trop Pediatr 2007;53:150-152.

10 Gökçe I, Alpay H, Biyikli N, Ozdemir N: Urinary tract pathogens and their antimicrobial resistance patterns in Turkish children. Pediatr Nephrol 2006;21:1327-1328.

11 Prelog M, Schiefecker D, Fille M, Wurzner R, Brunner A, Zimmerhackl LB: Febrile urinary tract infection in children: ampicillin and trimethoprim insufficient as empirical mono-therapy. Pediatr Nephrol 2008;23: 597-602.

12 Mårild S, Jodal U, Sandberg T: Ceftibuten versus trimethoprim-sulfamethoxazole for oral treatment of febrile urinary tract infection in children. Pediatr Nephrol 2009;24: 521-526.

13 Tseng MH, Lo WT, Lin WJ, Teng CS, Chu $\mathrm{ML}$, Wang CC: Changing trend in antimicrobial resistance of pediatric uropathogens in Taiwan. Pediatr Int 2008;50:797-800.
14 Al-Mardeni RI, Batarseh A, Omaish L, Shraideh M, Batarseh B, Unis N: Empirical treatment for pediatric urinary tract infection and resistance patterns of uropathogens, in Queen Alia Hospital and Prince A’isha Military Center - Jordan. Saudi J Kidney Dis Transpl 2009;20:135-139.

15 Akram M, Shahid M, Khan AU: Etiology and antimicrobial agent resistance patterns of community-acquired urinary tract infections in J N M C Hospital Aligarh, India. Ann Clin Microbiol Antimicrob 2007;6:4.

16 Hodson EM, Willis NS, Craig JC: Antibiotics for acute pyelonephritis in children. Cochrane Database Syst Rev 2007;4:CD003772.

17 Gupta K: Emerging antibiotic resistance in urinary tract pathogens. Infect Dis Clin North Am 2003;17:243-259.

18 Mandell LA, Peterson LR, Wise R, Hooper D, Low DE, Schaad UB, Klugman KP, Courvalin P: The battle against emerging antimicrobial agent resistance: should fluoroquinolones be used to treat children? Clin Infect Dis 2002;35:721-727.

19 Santo E, Salvador MM, Marin JM: Multidrug-resistant urinary tract isolates of Escherichia coli from Ribeirão Preto, São Paulo, Brazil. Braz J Infect Dis 2007;11:575-578.

20 Patterson JE: Extended spectrum beta-lactamases: a therapeutic dilemma. Pediatr Infect Dis J 2002;21:957-959.

-21 Borsari AG, Bucher B, Brazzola P, Simonetti GD, Dolina M, Bianchetti MG: Susceptibility of Escherichia coli strains isolated from outpatient children with community-acquired urinary tract infection in southern Switzerland. Clin Ther 2008;30:2090-2095. 